\title{
Fear of falling and associated factors among patients with peripheral vestibular hypofunction
}

\author{
Hee Seung Song' ${ }^{1}$ Hyun Jung Lee ${ }^{2, *}$ \\ 'Department of Nursing, College of Health \& Medical Science, Sangji University, Wonju, Korea \\ ${ }^{2}$ College of Nursing, Konyang University, Daejeon, Korea
}

The present study aimed to investigate the fear of falling and its associated factors among patients with vestibular hypofunction. We conducted a cross-sectional survey using structured questionnaires to evaluate fear of falling and activities of daily living among participants. Vestibular function was assessed via a rotary chair test. This study was conducted from January through March 2018. The subjects were 167 individuals older than 20 years who agreed to participate in this study. Data were analyzed using SPSS ver. 22.0. The significant variables associated with fear of falling were gender $(P=0.012)$, age $(P<0.001)$, marital status $(P=0.006)$, living alone $(P=0.020)$, having a job $(P<0.001)$, frequency of dizziness $(P=0.007)$, use of antivertigo medication $(P<0.001)$, and histo- ry of falling $(P<0.001)$. Fear of falling showed statistically significant positive correlations with activities of daily living $(r=0.82, P<0.001)$ and dizziness $(r=0.61, P<0.001)$. In the multiple regression analysis, age, gender, history of falling, and severity of dizziness explained $53.4 \%$ of the variance in fear of falling. The results of this study demonstrate that thoroughly assessing predictors making an impact on fear of falling in the initial assessment and the level of fear of falling needs to be addressed early to prevent sedentary behavior.

Keywords: Fear, Falls, Dizziness, Vestibular, Hypofunction

\section{INTRODUCTION}

A fall refers to an unintentional change in posture to the floor or a lower level (World Health Organization [WHO], 2019). Falls are hazardous incidents that may induce permanent disability by causing serious injuries, such as cerebral hemorrhage, mixed-mode fracture, and even death in more serious cases (Jahn, 2019; WHO, 2019). In particular, falls are more fatal among older people, as it may have a detrimental impact on their health and ability to live independently, thereby causing serious physical, mental, and economic problems. Thus, falls are perceived as an important healthcare issue and there is increasing interest in falls and their prevention. Old age may be a key contributor to falls (Callis, 2016; Jahn, 2019; WHO, 2019). However, in an assessment of vestibular function via the caloric test among community-dwelling older adults aged 65 years or older who had sustained at least two falls in the preceding year, Liston et al. (2014) reported that $80 \%$ of partici- pants had diminished vestibular function. Based on this, they pinpointed vestibular hypofunction as a major cause of falls. A diminished sense of balance, which is essential for standing and walking, is a major physical factor that induces falls. Vestibular hypofunction causes problems with the sense of balance, thereby triggering dizziness and difficulty standing or walking with the eyes open in the early days of symptom onset (Ganança et al., 2010).

Recently, the number of patients seeking medical attention with dizziness as their major complaint is increasing exponentially in Korea (Health Insurance Review and Assessment Service, 2019). Vestibular hypofunction is a major cause of dizziness, and failure to receive appropriate management early after onset may result in chronic dizziness. Continuous dizziness leads to a deterioration in one's quality of life by inducing physical problems such as falls and impaired activities of daily living (ADLs) as well as psychological problems such as fear of falling, anxiety, and depression (Lee and Choi-Kwon, 2009).
*Corresponding author: Hyun Jung Lee (ib https://orcid.org/0000-0002-8707-8630 College of Nursing, Konyang University, 158 Kwanjeodong-ro, Seo-gu,

Daejeon 35365, Korea

E-mail: dulzae94@gmail.com

Received: January 16, 2020 / Accepted: February 27, 2020
This is an Open Access article distributed under the terms of the Creative Commons Attribution Non-Commercial License (https://creativecommons.org/licenses/by-nc/4.0/) which permits unrestricted non-commercial use, distribution, and reproduction in any medium, provided the original work is properly cited. 
Vestibular function requires several weeks to months to be restored (Lacour et al., 2016). However, patients with dizziness with vestibular hypofunction were reported to have difficulty with ADLs, including standing, walking, and bathing, soon after onset. Due to dizziness experienced during motion and fear of falling, patients limit their physical activity and become passive to rehabilitation exercise that is essential for recovery. This, in turn, delays the vestibular compensation of the central nervous system (Lacour et al., 2016), thereby delaying the recovery of symptoms and ADL. Ultimately, this contributes to the persistence of dizziness, and the risk for falls lingers.

Only a handful of Korean studies has assessed falls among patients with dizziness. These studies have only identified the factors that affect dizziness using data of patients who have suffered a fall, examined the incidence of falls among patients with dizziness, and investigated fear of falling and impairment of ADLs (Lee and Lee, 2013; Lee and Choi-Kwon, 2009). Thus, the present study aimed to investigate the experiences of falls, fear of falling, and level of ADL among people with vestibular hypofunction objectively confirmed based on thorough medical examination and examined the relationships among these variables as well as the factors that impact fear of falling.

\section{MATERIALS AND METHODS}

\section{Study design}

The present cross-sectional study aimed to investigate fear of falling and ADL according to sociodemographic and health-related characteristics, correlations among the major variables, and factors that affect fear of falling among patients with peripheral vestibular hypofunction and dizziness.

\section{Participants}

Adults and older people aged 20 years or older who presented to a university hospital with dizziness as their major complaint and underwent vestibular function tests were recruited. The inclusion criteria were people within below normal range in at least three frequencies in the rotation chair test and people who could understand and respond to the items on the questionnaire. People who had been diagnosed with dizziness caused by a condition other than vestibular dysfunction (e.g., the central, psychogenic, and cervical types) and people who were then undergoing treatment for other medical or surgical acute diseases were excluded from this study. The minimum sample size required was estimated using G-Power 3.1.9 (Faul et al., 2007). With a significance level of
0.05 , power of $90 \%$, moderate effect size, and 12 predictors for multiple linear regression, the required sample size was calculated to be 157 . Considering potential withdrawals, 191 participants were recruited. After excluding 10 who could not complete the questionnaire due to severe dizziness, seven who did not consent to the study due to time, and seven who did not complete the questionnaire attentively, data from 167 participants were analyzed.

\section{Instruments}

\section{Sociodemographic characteristics}

Age, sex, marital status, cohabiting status, and employment status were surveyed as sociodemographic characteristics.

\section{Health-related characteristics}

Health-related characteristics including frequency of dizziness, history of falls due to dizziness, frequency of falls, and use of vestibular suppressants were surveyed using medical records and a self-reported questionnaire. In assessing the frequency of dizziness, dizziness was classified as transient, for cases that occurred and disappeared, intermittent, for cases that repeatedly occurred and disappeared, and continuous, for cases in which dizziness was persistent.

\section{Objective vestibular function}

Objective vestibular function was assessed via rotary chair testing (System 2000; Micromedical Technologies, Chatham, IL, USA), which was developed to measure ocular movement by stimulating the lateral semicircular cannal. In the rotary chair test, vestibular ocular reflex gain is recorded at 0.01, 0.04, and $0.16 \mathrm{~Hz}$, and patients with values below the normal range set by the manufacturer are diagnosed with vestibular hypofunction (Gimmon and Schubert, 2019).

\section{Dizziness}

Dizziness was quantified using the visual analogue scale (Toupet et al., 2011) from no dizziness (0) to extreme dizziness (10), with a higher score indicating severer dizziness.

\section{ADL impairment}

ADL was assessed using the vestibular disorders ADL (VADL) scale developed by Cohen et al. (2000) and adapted into Korean by Han et al. (2004). The VADL consists of 28 items rated on a 10-point scale, and the scale is divided into the functional, ambulation, and instrumental subscales. The functional subscale per- 
tains to basic ADLs, such as dressing and washing, while the ambulation subscale pertains to body movement, such as climbing the stairs and walking. The instrumental subscale pertains to household chores, work performance, and leisure activities. A higher score indicates greater difficulty with ADLs as a result of dizziness. We obtained permission for use from the original developer and researchers who adapted it into Korean. The reliability of the instrument as measured using Cronbach a was 0.97 in the study conducted by Han et al. (2004) and 0.95 in our study.

\section{Fear of falling}

Fear of falling was assessed using the Survey of Activities and Fear of Falling in the Elderly (SAFE) instrument developed by Lachman et al. (1998) and adapted into Korean through translation and back translation by Song et al. (2001). We obtained permission for use from the original developers and researchers who adapted it into Korean. The SAFE instrument is an 11-item tool rated on a four-point Likert scale (1: not afraid, 2: neutral, 3: slightly afraid, 4: very afraid), with a higher score indicating greater fear of falling. The reliability of the adapted instrument as measured using Cronbach a was 0.91 in the study conducted by Song et al. (2001) and 0.96 in our study.

\section{Ethical considerations}

The study protocol was approved by the Institutional Review Board (IRB No. 2017-12-003). Prior to data collection, we informed the participants of the purpose, method, and anticipated effects of the study and participants' rights to withdraw from the study at any time. Written informed consent was obtained from those who voluntarily expressed willingness to participate in the study.

\section{Data collection procedure}

The survey was conducted from December 9, 2017 to March 30, 2018 after obtaining IRB approval. Among patients who visited the outpatient department of ear, nose, and throat neurology at a university hospital with suspected peripheral vestibular hypofunction, those diagnosed with vestibular hypofunction based on vestibular function testing were surveyed at the dizziness clinic. The rotary chair test for the assessment of vestibular function was performed by two audiologists using the same equipment, and vestibular function test results and disease-related characteristics were obtained from the medical records. The survey took 10-15 min per participant. The researcher provided additional explanations when participants needed clarification and had difficulty re- sponding to the questions to minimize missing responses.

\section{Statistical analysis}

The data collected were analyzed by independent $t$-tests, analysis of variance, Pearson correlation analysis and multiple linear regression using IBM SPSS Statistics ver. 22.0 (IBM Co., Armonk, NY, USA).

\section{RESULTS}

\section{Sociodemographic and health-related characteristics}

The mean age of our participants was $57.55 \pm 13.50$ years, and there were 64 men (38.3\%) and 103 women (61.7\%). One hundred twenty-one participants $(72.5 \%)$ currently had a spouse while $46(27.5 \%)$ did not. While $130(77.8 \%)$ claimed to be living with family, 37 (22.2\%) stated that they did not live with family. One hundred two (61.1\%) were employed, and 65 (38.9\%) were not. Dizziness was persistent in 92 participants (55.1\%), intermittent in $56(33.5 \%)$, and transient in 19 (11.4\%). Sixty-one participants (36.5\%) were using vestibular suppressants and $106(63.5 \%)$ were not. The mean number of falls in the preceding 3 months was $0.73 \pm 2.52$ (Table 1).

\section{Fear of falling and ADL according to sociodemographic} and health-related characteristics

The fear of falling score was statistically significantly higher among women $(t=-3.78, P<0.001)$, people without a spouse $(t=$ -3.40, $P=0.001)$, people not living with family $(t=-3.09, P=$ $0.002)$, unemployed people $(t=-5.34, P<0.001)$, people who were currently taking medications $(t=2.69, P=0.008)$, and people with persistent dizziness $(F=6.69, P=0.002)$. The VADL score for impaired ADL was statistically significantly higher among women $(t=-2.58, P=0.012)$, people without a spouse $(t=-2.81, P=0.006)$, people not living with family $(t=-2.36, P=0.020)$, unemployed people $(t=-3.91, P<0.001)$, people who were currently taking medications $(t=4.07, P<0.001)$, and people with continuous dizziness $(F=5.19, P=0.007)$ (Table 1$)$.

\section{Correlations among severity of dizziness, ADL, and fear of falling}

The mean dizziness score was $5.68 \pm 2.24$ and the mean fear of falling score was $2.87 \pm 0.90$. The mean total score for VADL was $148.72 \pm 2.10$ and the mean rating was $5.42 \pm 2.59$ (Table 2). There were significantly strong positive correlations between dizziness and VADL score $(r=0.67, P<0.001)$, dizziness and fear of falling 
Table 1. Fear of falling and activities of daily living (ADLs) according to demographic and clinical characteristics ( $n=167)$

\begin{tabular}{|c|c|c|c|c|c|}
\hline \multirow{2}{*}{ Variable } & \multirow{2}{*}{ №. (\%) } & \multicolumn{2}{|c|}{ Fear of falling } & \multicolumn{2}{|c|}{ ADL } \\
\hline & & Mean \pm SD & tor $F(P)$ & Mean \pm SD & tor $F(P)$ \\
\hline \multicolumn{6}{|l|}{ Gender } \\
\hline Men & $64(38.3)$ & $2.54 \pm 0.96$ & -3.78 & $4.75 \pm 2.92$ & -2.55 \\
\hline Women & $103(61.7)$ & $3.08 \pm 0.79$ & $(<0.001)$ & $5.84 \pm 2.27$ & $(0.012)$ \\
\hline \multicolumn{6}{|l|}{ Age (yr) } \\
\hline $20-34^{\mathrm{a})}$ & $8(4.8)$ & $2.02 \pm 0.86$ & 9.88 & $3.36 \pm 2.02$ & 8.51 \\
\hline $35-49^{b)}$ & $37(22.2)$ & $2.64 \pm 0.89$ & $(<0.001)$ & $4.67 \pm 2.64$ & $(<0.001)$ \\
\hline $50-64^{c)}$ & $73(43.7)$ & $2.75 \pm 0.86$ & $a, b, c<d$ & $5.12 \pm 2.50$ & $a, b, c<d$ \\
\hline$\geq 65^{\mathrm{dl}}$ & $49(29.3)$ & $3.36 \pm 0.73$ & & $6.77 \pm 2.20$ & \\
\hline \multicolumn{6}{|l|}{ Marital status } \\
\hline Yes & $121(72.5)$ & $2.73 \pm 0.88$ & -3.40 & $5.08 \pm 2.55$ & -2.81 \\
\hline No (divorced, bereaved, and single) & $46(27.5)$ & $3.24 \pm 0.82$ & $(<0.001)$ & $6.32 \pm 2.49$ & $(0.006)$ \\
\hline \multicolumn{6}{|l|}{ Living alone } \\
\hline Yes & 130 (77.8) & $2.76 \pm 0.90$ & -3.09 & $5.18 \pm 2.55$ & -2.36 \\
\hline No & $37(22.2)$ & $3.26 \pm 0.78$ & $(0.002)$ & $6.30 \pm 2.57$ & $(0.020)$ \\
\hline \multicolumn{6}{|l|}{ Employment status } \\
\hline Yes & $102(61.1)$ & $2.61 \pm 0.91$ & -5.34 & $4.83 \pm 2.56$ & -3.91 \\
\hline No & $65(38.9)$ & $3.28 \pm 0.71$ & $(<0.001)$ & $6.37 \pm 2.35$ & $(<0.001)$ \\
\hline \multicolumn{6}{|l|}{ Frequency of dizziness } \\
\hline Persistent ${ }^{\text {al }}$ & $92(55.1)$ & $3.09 \pm 0.85$ & 6.69 & $5.99 \pm 2.63$ & 5.19 \\
\hline Intermittent $t^{b)}$ & $19(11.4)$ & $2.56 \pm 0.95$ & $(0.002)$ & $4.68 \pm 2.50$ & $(0.007)$ \\
\hline Transientc) & $56(33.5)$ & $2.61 \pm 0.86$ & $c<a$ & $4.74 \pm 2.35$ & $c<a$ \\
\hline \multicolumn{6}{|l|}{ Use of antivertigo medication } \\
\hline Yes & $61(36.5)$ & $3.11 \pm 0.88$ & 2.69 & $6.45 \pm 2.43$ & 4.07 \\
\hline No & $106(63.5)$ & $2.73 \pm 0.88$ & $(0.008)$ & $4.83 \pm 2.50$ & $(<0.001)$ \\
\hline \multicolumn{6}{|l|}{ History of falling } \\
\hline Yes & $63(37.7)$ & $3.48 \pm 0.63$ & 8.585 & $7.11 \pm 2.23$ & 7.615 \\
\hline No & $104(62.3)$ & $2.50 \pm 0.83$ & $(<0.001)$ & $4.40 \pm 2.23$ & $(<0.001)$ \\
\hline
\end{tabular}

$\mathrm{SD}$, standard deviation.

Table 2. Correlation among fear of falling, dizziness, and activities of daily living (ADLs) ( $n=167)$

\begin{tabular}{lccc}
\hline Variable & Fear of falling & Dizziness & ADL \\
\hline Fear of falling & 1.00 & - & - \\
Dizziness & $0.61(<0.001)$ & 1.00 & - \\
ADL & $0.82(<0.001)$ & $0.67(<0.001)$ & 1.00
\end{tabular}

Values are presented as $r(P$-value $)$.

$(r=0.61, P<0.001)$, and fear of falling and VADL score $(r=0.82$, $P<0.001$ ) (Table 2).

\section{Factors that impact fear of falling}

To identify factors that impact fear of falling, the variables that were significant in the univariate analysis (namely sex, spouse, cohabitation with family, employment status, current use of medi- cation, and frequency of dizziness) were dummy coded, and age, number of falls in the past 3 months, and current level of dizziness were included as independent variables. ADL was excluded from the independent variables due to the possibility of collinearity as a result of strong correlation $(r=0.821, P<0.001)$. The absence of multicollinearity was confirmed, as the tolerance limits among the independent variables ranged from 0.519-0.919 and exceeded the cutoff of 0.1 , and the variance inflation factor ranged from 1.133-1.926, all below the cutoff value of 10 . The results showed that fear of falling increased with increasing age $(\beta=0.27, P<$ $0.001)$, among women $(\beta=0.15, P=0.016)$, among those with a history of falls $(\beta=0.11, P=0.045)$, and among those who currently had severe dizziness $(\beta=0.51, P<0.001)$. The explanatory power of the model was $53.4 \%$, and the model was statistically significant $(F=20.04, P<0.001)$ (Table 3). 
Table 3. Factors influencing fear of falling $(n=167)$

\begin{tabular}{lccccr}
\hline Variable & B & SE & $\beta$ & $t$ & $P$-value \\
\hline Age & 0.02 & 0.01 & 0.27 & 3.70 & $<0.001$ \\
Gender $^{\text {a) }}$ & 0.27 & 0.11 & 0.15 & 2.44 & 0.016 \\
History of fallinga) $^{\text {a) }}$ & 0.04 & 0.02 & 0.11 & 2.02 & 0.045 \\
Dizziness & 0.21 & 0.02 & 0.51 & 9.28 & $<0.001$
\end{tabular}

SE, standard error.

$R^{2}=0.562$, adjusted $R^{2}=0.534, F=20.04, P<0.001$, Durbin-Watson statistic $=1.79$.

${ }^{a}$ Dummy variable: gender $(0=$ female, $1=$ male), history of falling $(0=y e s, 1=n o)$.

\section{DISCUSSION}

In the assessment of the risk of falls, people with reduced vestibular function and dizziness are considered to be at high risk (WHO, 2019). Patients with dizziness with vestibular hypofunction limit themselves from engaging in ADLs and rehabilitative exercises due to a fear of falling, which in turn hinders vestibular compensation, ultimately further elevating the risk of fall and reducing their quality of life (Jahn, 2019). Thus, the present study aimed to investigate the fear of falling and its influencing factors among patients with dizziness secondary to vestibular hypofunction.

In terms of participants' demographic characteristics, fear of falling was significantly more common among women, older persons, unemployed persons living alone, and people with a history of falls (Table 1). The high fear of falling among older people and those living alone are in line with the results of a study on fear of falling among patients with rheumatoid arthritis (Kim, 2007). Although the said study only involved older women and thus could not examine sex-specific differences, previous studies on community-dwelling older people (Song et al., 2001) found that fear of falling was significantly more common among women, older people, and those living alone, as found in our study. This result supports previous findings that weakened social support, which alleviates and helps overcome psychological fear, has an adverse impact on fear of falling.

In terms of health-related characteristics, fear of falling significantly differed according to persistence of dizziness, use of vestibular suppressants, and history of falls (Table 1). Persistent dizziness and the use of vestibular suppressants indicate the severity of the symptoms, and the high severity of symptoms seems to have contributed to the fear of falling. Moreover, we found that people with a history of fall had a greater fear of falling. Previous studies also reported that a history of fall not only induces physical injuries but also has an adverse psychological impact, and that it may serve as a major predictor of future falls (Jahn, 2019; Kim, 2007). In our study, fear of falling was significantly greater among those with a history of fall, and this suggests that fall prevention activities should be more focused on patients with dizziness with vestibular hypofunction who have sustained falls in the past.

In our study, $37.7 \%$ of the participants stated that they had a previous history of fall. A sense of balance is known to be the greatest contributor to falls (Jahn, 2019) and a problem with balance as a result of vestibular hypofunction causes a serious problem with standing and walking. In other words, the risk of falls increases with the increasing degree of vestibular hypofunction. Liston et al. (2014) reported that $80 \%$ of community-dwelling older people who had at least two falls a year had diminished vestibular function as diagnosed based on caloric testing. A Korean study that reported that 93 out of 148 (62.8\%) older women aged 65 years or older living alone with a history of falls suffered from dizziness (Lee and Lee, 2013) support this hypothesis. This suggests that studies on falls consider assessment of vestibular function, which impacts participants' sense of balance especially on elderly.

In our study, fear of falling was strongly positively correlated with impaired ADL and level of dizziness (Table 2). That is, fear of falling increased with greater impaired ADL and dizziness. This shows that fear of falling is closely related to limited physical activity, which suggests that psychological factors among participants served as an obstacle to active involvement.

Multiple regression identified severity of dizziness, age, sex, and history of falls as factors that impact fear of falling (Table 3). Although we cannot compare our results to those of previous studies due to the lack of studies that assessed factors that affect fear of falling among patients with vestibular hypofunction, individualized strategies to reduce fear of falling should be devised for vestibular rehabilitation exercise among female patients with severe dizziness and a history of falls.

\section{CONFLICT OF INTEREST}

No potential conflict of interest relevant to this article was reported.

\section{ACKNOWLEDGMENTS}

The authors acknowledge the Department of Otolaryngology-Head \& Neck Surgery, Dankook University Hospital. 


\section{REFERENCES}

Callis N. Falls prevention: identification of predictive fall risk factors. Appl Nurs Res 2016;29:53-58.

Cohen HS, Kimball KT, Adams AS. Application of the vestibular disorders activities of daily living scale. Laryngoscope 2000;110:1204-1209.

Faul F, Erdfelder E, Lang AG, Buchner A. G*Power 3: a flexible statistical power analysis program for the social, behavioral, and biomedical sciences. Behav Res Methods 2007;39:175-191.

Ganança FF, Gazzola JM, Ganança CF, Caovilla HH, Ganança MM, Cruz OL. Elderly falls associated with benign paroxysmal positional vertigo. Braz J Otorhinolaryngol 2010;76:113-120.

Gimmon Y, Schubert MC. Vestibular testing-rotary chair and dynamic visual acuity tests. Adv Otorhinolaryngol 2019;82:39-46.

Han GC, Lee EJ, Lee JH, Park SN, Lee HY, Jeon EJ. The study of standardization for a Korean adaptation of self-report measures of dizziness. Res Vestib Sci 2004;3:307-325.

Health Insurance Review and Assessment Service. Cause of dizziness. [Internet] Seoul (Korea): Health Insurance Review and Assessment Service Korea; 2019 [cited 2019 Jan 10]. Available from: http://www. hira.or.kr/dummy.do?pgmid=HIRAA020041000000\&cmsurl=/cms/ notice/02/131730113390.html.

Jahn K. The aging vestibular system: dizziness and imbalance in the elderly. Adv Otorhinolaryngol 2019;82:143-149.

Kim JS. Influencing factors for fear of falling in degenerative arthritis patients. Taehan Kanho Hakhoe Chi 2007;37:1184-1192.
Lachman ME, Howland J, Tennstedt S, Jette A, Assmann S, Peterson EW. Fear of falling and activity restriction: the survey of activities and fear of falling in the elderly (SAFE). J Gerontol B Psychol Sci Soc Sci 1998; 53:P43-50.

Lacour M, Helmchen C, Vidal PP. Vestibular compensation: the neurootologist's best friend. J Neurol 2016;263 Suppl 1:S54-64.

Lee HJ, Choi-Kwon S. Quality of life and the related factors in patients with dizziness. J Korean Acad Nurs 2009;39:751-758.

Lee MS, Lee YB. Fear of falling and related factors in elderly living alone based on fall experience. J Agric Med Community Health 2013;38:243256.

Liston MB, Bamiou DE, Martin F, Hopper A, Koohi N, Luxon L, Pavlou M. Peripheral vestibular dysfunction is prevalent in older adults experiencing multiple non-syncopal falls versus age-matched non-fallers: a pilot study. Age Ageing 2014;43:38-43.

Song KY, Moon JS, Kang SS, Choi JH. The survey of activities and fear of falling in the community dwelling elderly. J Korean Public Health Nurs 2001;15:324-333.

Toupet M, Ferrary E, Grayeli AB. Visual analog scale to assess vertigo and dizziness after repositioning maneuvers for benign paroxysmal positional vertigo. J Vestib Res 2011;21:235-241.

World Health Organization. Global report on falls prevention in older age [Internet]. Geneva (Switzerland): World Health Organization; c2019 [cited 2019 Jan 10]. Available from: https://extranet.who.int/agefriendlyworld/global-report-on-falls-prevention-in-older-age/. 